\title{
Integrated analysis identifies low microRNA-215 expression as associated with a poor prognosis of patients with colorectal cancer through the IK $\beta-\alpha$ signaling pathway
}

\author{
Zhuo Wang ${ }^{1,2,3}$, Xin Jiang ${ }^{3,4}$, Qing Li $^{1,3}$, Yufen Jin ${ }^{3,4}$, Xingxiang Liu ${ }^{1,3}$, Fang Wang ${ }^{4}$, Yong Mao ${ }^{1,3}$, \\ Dong Hua ${ }^{1,3}$
}

${ }^{1}$ Department of Oncology, The Second Affiliated Hospital of Soochow University, Soochow, China; ${ }^{2}$ Department of Geriatric, Wuxi People's Hospital Affiliated to Nanjing Medical University, Wuxi, China; ${ }^{3}$ Department of Oncology, Affiliated Hospital of Jiangnan University, Wuxi, China; ${ }^{4}$ Department of Public Health and Preventive Medicine, Jiangnan University School of Wuxi Medicine, Wuxi, China

Contributions: (I) Conception and design: Z Wang, Q Li, Y Mao; (II) Administrative support: Y Mao, D Hua; (III) Provision of study materials or patients: Q Li, Y Jin; (IV) Collection and assembly of data: X Jiang, X Liu; (V) Data analysis and interpretation: X Liu, F Wang; (VI) Manuscript writing: All authors; (VII) Final approval of manuscript: All authors.

Correspondence to: Professor Dong Hua, MD, PhD. Department of Oncology, The Second Affiliated Hospital of Soochow University, Soochow, China. Email: wx89211@163.com; Professor Yong Mao, MD, PhD. Department of Oncology, Affiliated Hospital of Jiangnan University, Wuxi, China. Email: mydoctorwx@aliyun.com.

Background: miRNA expression data on colorectal cancer (CRC) are constantly updated. Therefore, integrated analysis of these datasets prior to experiments is necessary in translational medicine and oncology research. Abnormal low expression of hsa-miRNA-215-5p (miR-215) is detected in several cancer types, but the role of miR-215 in CRC remains unclear. Therefore, the aim of this work was to identify the expression and role of miR-215 involved in CRC.

Methods: An integrated analysis of 4 sets of miRNA microarray data of CRC in GEO was implemented. The low expression of miR-215 in CRC was confirmed by TCGA datasets. In addition, frozen tissue and paired formalin-fixed paraffin-embedded samples were collected from 214 CRC patients who underwent CRC surgery at the Affiliated Hospital of Jiangnan University, China, and used as an independent clinical validation study. Furthermore, colon cancer cells HCT116 and SW480 transfected with miR-215 mimic/ inhibitor were used to evaluate its mechanism of action and to perform experiments to confirm our results obtained from human samples.

Results: CRC patients with a decreased miR-215 expression in adenocarcinoma tissues had a significantly poor prognosis with lower cumulative survival as revealed by the TCGA-COAD dataset. In our 214 CRC patients cohort study this result was confirmed and it was also found that low miR-215 expression was inversely correlated with the expression of IK $\beta-\alpha$. Downregulation of miR-215 in HCT116 and SW480 cells resulted in an upregulation of TRAF5 and TAK1 protein expression, and interfered with IK $\beta$ - $\alpha$ protein expression. Furthermore, with the inhibition of miR-215, important Epithelial-Mesenchymal Transition (EMT) biomarker proteins were significantly upregulated in HCT116 and SW480 cells. Moreover, an inhibition was obtained using miR-215-mimic.

Conclusions: Our integrated microRNA dataset approach identified miR-215 as an independent factor associated with the prognosis of CRC patients. In addition, our results demonstrated that miR-215 might be considered as a potential biomarker for poor prognosis in CRC patients and its role as a potent suppressor of IK $\beta-\alpha$ and TRAF5.

Keywords: hsa-miRNA-215-5p (miR-215); TRAF5; colorectal cancer (CRC); prognosis; biomarker

Submitted Nov 07, 2019. Accepted for publication Apr 14, 2020.

doi: $10.21037 /$ tcr-19-2424

View this article at: http://dx.doi.org/10.21037/tcr-19-2424 


\section{Introduction}

Colorectal cancer (CRC) is one of the most common malignancies worldwide, with the world's third highest incidence, and the fourth highest malignancy (1). In China, CRC has an increasing incidence due to changes in the diet and lifestyle (2). CRC is a multi-pathway tumor and its progression and pathological properties are influenced by a variety of factors including the genetic one, environment and lifestyle (3).

MicroRNAs (miRNAs) are a class of non-coding small RNAs that regulate gene expression at a posttranscriptional level $(4,5)$. An aberrant expression of several miRNAs is present in a wide range of human cancer types, including CRC $(6,7)$. As a tumor suppressor, miR-215 is downregulated in hepatocellular carcinoma, cervical cancer, and thyroid cancer (8-10). These studies indicate that the decreased expression of miR-215 is closely associated with tumor growth and metastasis $(11,12)$, leading to tumorigenesis and predicting high malignancy and poor prognosis (13). However, the correlation between miR215 and malignant CRC progression is currently less investigated. Thus, miR-215 role in CRC is still unclear.

Therefore, in this work an integrated analysis was performed. Low expression of some miRNAs was detected on microRNA sequencing data in colorectal adenocarcinoma tissue and adjacent normal tissue in Gene Expression Omnibus (GEO) datasets (14). The aberrant low expression of these miRNAs was confirmed in the TCGA-COAD, revealing that miR-215 expression was low in patients with poor survival. Thus, it might play an important role in the development and progression of CRC. Under miR-215 low expression after its inhibition in colon cancer cells, functional enrichment analysis of differential gene transcription levels suggested cytokine interaction (15). Our experiments using two colon cancer cell lines, HCT116 and SW480, revealed that IK $\beta-\alpha$, TRAF5 and TAK1 expression was upregulated with the inhibition of miR-215 using miR-215-mimic. EMT important biomarker proteins including $\mathrm{N}$-cadherin, vimentin and MMP7 were also significantly upregulated in HCT116 and SW480 after miR-215 inhibition. Experiments on specimens from intestinal cancer patients after surgery also confirmed these results. Thus, our results offer a plausible mechanism for the tumor-suppressing function of miR-215, through its action on TRAF5 and IK $\beta-\alpha$, potentially having an important impact on the occurrence and development of CRC and poor prognosis of these patients.

\section{Methods}

\section{Data collection, preprocessing and miRNA datasets integrated analysis}

Public miRNA microarray repositories were searched in the NCBI GEO. The inclusion criteria were the following: (I) dataset of colorectal adenocarcinoma tissue and adjacent normal tissue (16); (II) sequences performed using the Illumina Hiseq platform; (III) Dataset of studies after 2012, with a number of specimens not less than 20 (17). The soft file or expression matrix file of the original result was downloaded. Next, a validation study of the results of these published datasets was performed in our present study. The exclusion criteria were the following: (I) duplicate reports uploaded by the same research institution; (II) documents involving non-human genome-wide file (18); (III) datasets individually normalized on the base-2 logarithm by Robust Multi-Array Average (RMA) and Linear Models for Microarray (LIMMA) package (19). For gene id conversion of all miRNAs, the gene name was referred to the miRBase at http://www.mirbase.org/ for matching annotations (20), version October 2018. Finally, the GEO datasets included in our study were the following: accession numbers GSE48267, GSE38389, GSE41655, and GSE49246.

The microRNA dataset in The Cancer Genome Atlas (TCGA) was used for results validation. TCGA-COAD miRNA data, RNA-seq data and related clinical data were downloaded from https://xenabrowser.net/ (21), and the data were updated to November 2018. To assess the prospective functions of the genes influenced by has-miR-215, the Kyoto Encyclopedia of Genes and Genomes (KEGG) was employed using the $\mathrm{R}$ software Bioconductor packages.

\section{Patients' samples}

A total of 214 CRC patients who underwent CRC surgery from January to December 2008 in the Oncology Department, Affiliated Hospital of Jiangnan University, Wuxi, China, were enrolled in the present study. Tissues from patients with pathologically confirmed tumors were snapfrozen and stored at $-80^{\circ} \mathrm{C}$. Patients' clinical characteristics are shown in Table 1. The last follow-up was the end of November 2013. Formalin-fixed paraffin-embedded tissue specimens from the same patients enrolled in this study were also collected based on the patient's medical records. The clinicopathological characteristics analyzed included patient gender, age, tumor size, histologic grade, primary tumor, nodal metastasis, pathological stage, vascular invasion, neural 
Table 1 Baseline characteristics of patients

\begin{tabular}{|c|c|c|c|c|}
\hline Clinical parameters & \multicolumn{2}{|c|}{ miR-215 expression } & OR $(95 \% \mathrm{Cl})$ & $P$ value \\
\hline Gender & & & & 0.493 \\
\hline Female $(n=103)$ & $65(51.4)$ & $38(51.4)$ & $1.22(0.69-2.14)$ & \\
\hline Male $(n=111)$ & $75(53.6)$ & $36(48.6)$ & 1.00 & \\
\hline$<63(\mathrm{n}=105)$ & $71(50.7)$ & $34(45.9)$ & $0.83(0.47-1.45)$ & \\
\hline$\geq 63(n=109)$ & $69(49.3)$ & $40(54.1)$ & 1.00 & \\
\hline Tumor location & & & & 0.303 \\
\hline Colon $(n=98)$ & $121(86.4)$ & $60(81.1)$ & $0.86(0.63-1.18)$ & \\
\hline III stage $(n=108)$ & $73(52.1)$ & $25(33.8)$ & $2.04(1.03-4.04)$ & \\
\hline III-IV stage $(\mathrm{n}=77)$ & $67(47.9)$ & $49(66.2)$ & 1.00 & \\
\hline Tumor (T) status & & & & 0.105 \\
\hline $\mathrm{T} 1+\mathrm{T} 2(\mathrm{n}=42)$ & $23(16.4)$ & $19(25.7)$ & $1.75(0.88-3.49)$ & \\
\hline T3+T4 $(n=172)$ & $117(83.6)$ & $55(74.3)$ & 1.00 & \\
\hline Nodal $(\mathrm{N})$ metastasis & & & & 0.771 \\
\hline N0 $(n=128)$ & $85(60.7)$ & $43(28.1)$ & $0.90(0.51-1.59)$ & \\
\hline $\mathrm{N} 1+\mathrm{N} 2(\mathrm{n}=86)$ & 55 (39.3) & $31(41.9)$ & 1.00 & \\
\hline
\end{tabular}

*, significantly different by the Pearson chi-squared test, $\mathrm{P}<0.05$ was considered statistically significant.

invasion, and lymphatic invasion. All patients enrolled in this study provided written informed consent to the use of their tissues, and this study was approved by the Ethics Committee Affiliated of Hospital of Jiangnan University, China (No. 20180642). The study was conducted in accordance with the Declaration of Helsinki (as revised in 2013).

\section{Real-time PCR}

Total RNA was extracted from tumor tissues using TRIzol (Invitrogen) according to the manufacturer's instructions. Total RNA with OD260/OD280 $=1.8-2.0$ was used. cDNA was obtained using the High-Capacity cDNA Reverse Transcription Kit (Applied Biosystems, Foster City, CA) according to the manufacturer's instructions.
miR-215 expression was detected by real-time PCR using SYBRGreen PCR Master Mix (Applied Biosystems, Foster City, CA) according to the manufacturer's instructions. Primers targeting miR-215 and U6 were used as previously described. The relative miR-215 expression was calculated using the $2^{-\Delta \Delta \mathrm{Ct}}$ method with $\mathrm{U} 6$ as the endogenous control.

\section{Immunobistochemical staining}

Immunohistochemistry was performed on the $4 \mu \mathrm{m}$ thick tissue sections from the formalin-fixed paraffinembedded tissue specimens of the CRC patients. After soaking in xylene three times for five minutes each time, and the subsequent passages in gradient alcohol, sections were placed in distilled water for 5 minutes. Next, antigen 
retrieval was performed using a sodium citrate solution for 20 minutes in boiling water. Endogenous peroxidase was blocked by incubating the sections with hydrogen peroxide for 5 minutes. Sections were then incubated overnight at $4{ }^{\circ} \mathrm{C}$ with primary Monoclonal rabbit antibodies against TRAF5 (1:150, Wuhan, China) and IK $\beta-\alpha(1: 200$, Wuhan, China). Sections were washed, incubated with the amplification agent and polymerase (reagent A, GTVisionTM III Kit supply, Shanghai, China), and then stained with 3,3'-diaminobenzidine (DAB, reagent $B$ and C, GTVisionTM III Kit supply, Shanghai, China). Sections were counterstained with hematoxylin. The negative control was treated in an identical manner using PBS instead of the primary antibody. A score from 0 to 4 was associated to each section according to the percentage of positively-stained cells in the entire section (no positive staining or $\leq 5 \%=0$; $6-25 \%$ positive $=1 ; 26-50 \%$ positive $=2 ; 51-75 \%$ positive $=3$; and $76-100 \%$ positive $=4$ ). The scoring intensity was determined as previously described: no staining $=0$, weak staining $=1$, moderate staining $=2$, strong staining $=3(22)$. Each case had two cores that were observed in two different fields with one core viewed under high magnification (200x).

\section{Cell culture and transfection of miR-215 mimic/inbibitor}

Two human CRC cell lines, HCT116 and SW480 were purchased from the American Type Culture Collection (ATCC, Manassas, VA, USA). These two cell lines have different miR-215 expression levels compared to other CRC cell lines. Cells were cultured in DMEM medium (Hyclone, Logan City, USA) containing 10\% fetal calf serum (Gibco, Carlsbad, USA), 1\% streptomycin (100 U/mL) -penicillin (100 U/mL) (Byotime, Nantong, China), and maintained in a humidified atmosphere of $5 \% \mathrm{CO}_{2}$ at $37^{\circ} \mathrm{C}$. miR-215 mimic and negative control mimic-NC and miR-215 inhibitor and negative control inhibitor-NC were purchased from Gene Pharma (Shanghai, China). Both cells were plated at $5 \times 10^{5}$ cells per $\mathrm{ml}$ in 6 -well plates and transfected with the mimic or inhibitors mentioned above using Lipofectamine 3000 (Invitrogen, Carlsbad, USA) according to the manufacturer's instructions. Cells were washed with PBS (pH 7.4) before performing transient transfection with $100 \mathrm{nM}$ miR-215 mimic and mimic-NC or miR-215 inhibitor and inhibitor-NC.

\section{Western blotting}

Total proteins were extracted from both cell types using
RIPA lysis buffer containing protease-phosphatase inhibitors (ab201119, abcam, the UK), and PMSF (Solarbio, Beijing, China) and placed on ice for $15 \mathrm{~min}$. Proteins were separated by $10 \%$ SDS-PAGE and transferred to PVDF membranes (Merck Millipore, Darmstadt, Germany). Membranes were blocked with $5 \%$ skim milk in trisbuffered saline containing $0.2 \%$ Tween-20 (TBST) for $40 \mathrm{~min}$ and were then treated with monoclonal rabbit antibodies anti TRAF5 (1:400, ProteinTech, Wuhan, China), IK $\beta-\alpha$ (1:200, ProteinTech, Wuhan, China), $\mathrm{N}$-cadherin (1:200, Beyotime, Nantong, China), Vimentin (1:200, Beyotime, Nantong, China) and MMP7 (1:200, Beyotime, Nantong, China), and monoclonal mouse GAPDH (1:1,000, ThermoFisher, Carlsbad, USA) and incubated overnight at $4{ }^{\circ} \mathrm{C}$. After washing three times with TBST for $15 \mathrm{~min}$, membranes were incubated with the secondary antibody for $40 \mathrm{~min}$ at room temperature. After washing three times with TBST for $10 \mathrm{~min}$, the immunoreactive bands were visualized using ECL Plus kit (Beyotime, Nantong, China) and the imaging analyzer (Biorad, USA) was used for observation and image acquisition. GAPDH was used as a loading control.

\section{Statistical analysis}

Statistical analysis was performed using IBM SPSS STATS (version 20) and R (for Windows version 3.6.0) software. As regard miR-215 TCGA data results and real-time PCR results on tissue samples, the median was used as the cutoff value to distinguish miR-215 high expression and low expression samples. The relationship between miR215 expression and clinical parameters was performed by univariate and multivariate forward stepwise logistic regression analysis. Survival curves were plotted using the Kaplan-Meier method and compared using the log-rank test. Univariate and multivariate survival analyses were performed by Cox regression model. A receiver operating characteristic (ROC) curve was set up by a logistic regression model to predict the effect of multivariate factors on the prognostic outcome of CRC patients. A P value less than 0.05 was considered statistically significant.

\section{Results}

\section{Integrated analysis of CRC miRNAs expression datasets}

Based on our inclusion and exclusion criteria, data on the expression of miRNAs were collected in 203 cancer tissues 
and 187 adjacent normal tissues. The $\log 2 \mathrm{FC} \geq|0.3|$ and the $\mathrm{P}$ value $<0.0001$ were used to screen for differentially expressed miRNAs and the thermograms were plotted, as shown in Figure 1A,B,C,D. hsa-miR-215, hsa-miR-378, hsa-miR-1, hsa-miR-29, hsa-miR-497, and hsa-miR-145 were downregulated in three or more of the four datasets related to cancer tissues. The data of the four datasets were normalized and the statistical analysis was performed (Figure 1E). hsa-miR-215, hsa-miR-378, hsa-miR-1, hsamiR-29, and hsa-miR-145 expression in tumor tissues was significantly lower than that in the adjacent normal colon epithelial tissue.

To further confirm the effect reported in the literature regarding the above miRNAs on the prognosis of patients with CRC, miRNAs dataset and clinical parameters were obtained from the COAD dataset of TCGA. Based on the expression of all the 6 miRNAs, the Kaplan-Meier method was used to perform the survival analysis, and the survival curve was plotted as shown in Figure $2 A, B, C, D, E, F$. Patients with low miR-215 expression in the tumor had a significantly poor survival than those with high expression $(\mathrm{P}<0.05)$.

According to the high and low miR-215 expression subgroups, the mRNA of all genes present in the TCGACOAD was grouped according to their differential expression. The results showed that the expression of TRAF5 in the low expression miR-215 group was higher than that in the high expression miR-215 group. TRAF5 mRNA expression in the low expression miR-215 group was subjected to KEGG functional enrichment analysis. The results are shown in Figure $2 G, H, I$, in which the cytokinecytokine interaction signaling pathway was upregulated, suggesting that the abnormal miR-215 low expression might affect the development of CRC by affecting the tumor microenvironment including cytokines.

\section{miR-215 expression in CRC tissues, clinical parameters and IKB- $\alpha$ expression in CRC patients}

Combined with the expression of miR-215 and miR-145 in the TCGA dataset and poor prognosis, the ROC curve was plotted based on the FPKM values of miR-215 and miR145 and the 5 -year survival rate of the patients, as shown in Figure 2G,H.

miR-215 expression was divided into high or low level categories according to the median of the expression outcome. Therefore, the number of patients with high and low miR-215 expression was 105 and 109, respectively.
Table 1 is a table showing the statistical analysis between the baseline data of the enrolled patients and the expression of miR-215. miR-215 expression was statistically correlated with the TNM stage of patients with CRC. In addition, patients with stage III and IV CRC were more likely to have low miR-215 expression, while patients with stage I and II were more likely to have high miR-215 expression (chi-square $=6.537, \mathrm{P}=0.013$ ). TRAF5 and IK $\beta$ - $\alpha$ protein expression was detected in TCGA as shown in Figure $3 \mathrm{~A}$. The binarization of the TRAF 5 and IK $\beta-\alpha$ mRNA expression was performed with the median high and low expression. In addition, chi-square analysis was performed comparing the expression of miR-215 with TRAF5 and IK $\beta-\alpha$ protein expression (by immunohistochemistry) (Table 2). In the patient cohort, low miR-215 expression was significantly associated with high TRAF5 and IK $\beta-\alpha$ expression, with a $\mathrm{P}$ value of 0.020 for TRAF and $\mathrm{P}<0.001$ for IK $\beta-\alpha$.

\section{miR-215 downregulation was associated with of IK $\beta-\alpha$ and EMT upregulation}

The linear regression analysis of miR-215 and TRAF, TAK1 and IK $\beta-\alpha$ expression was performed based on mRNAseq and miRNA data, as shown in Figure $3 A, B$. The database analysis of GEO and TCGA and the results of our tissue sections were further verified using two intestinal cancer cell lines. After HCT116 cell line transfection with miR-215 mimic, the results showed that TRAF5 and IK $\beta-\alpha$ expression was significantly higher than their expression in the negative control group in the HCT116 cell line (Figure 3C,D). Same results were also obtained in the SW480 cell line after treatment with miR-215 inhibitor (Figure $3 E, F$ ). After transfection with miR-215 mimic into HCT116 cells, $\mathrm{N}$-cadherin $(\mathrm{P}<0.05)$, vimentin $(\mathrm{P}<0.01)$ and MMP7 $(\mathrm{P}<0.01)$ protein expression was significantly decreased compared to their expression in the HCT116-NC. Similar results were obtained in SW480 cells; after transfection of miR-215-inhibitor into SW480 cells, $\mathrm{N}$-cadherin $(\mathrm{P}<0.01)$, vimentin $(\mathrm{P}<0.05)$ and $M M P 7(\mathrm{P}<0.01)$ protein expression was significantly increased compared to their expression in SW480-miR-215-NC. This, by interfering with miR-215 expression in CRC cell, changes in proteins belonging to the Wnt- $\beta$-catenin and EMT pathway were observed.

\section{Prognostic value of miR-215 expression in CRC patients}

The results of cell-mediated regulation resulted in the 
A

GSE48267

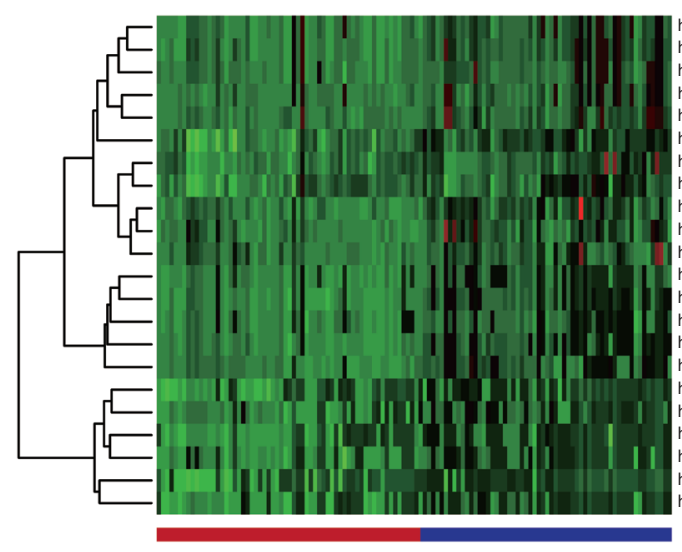

C

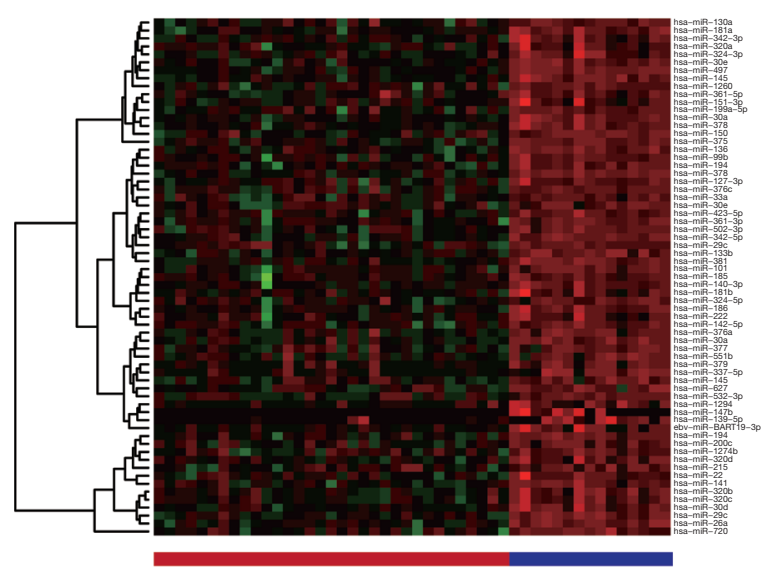

GSE41655
B
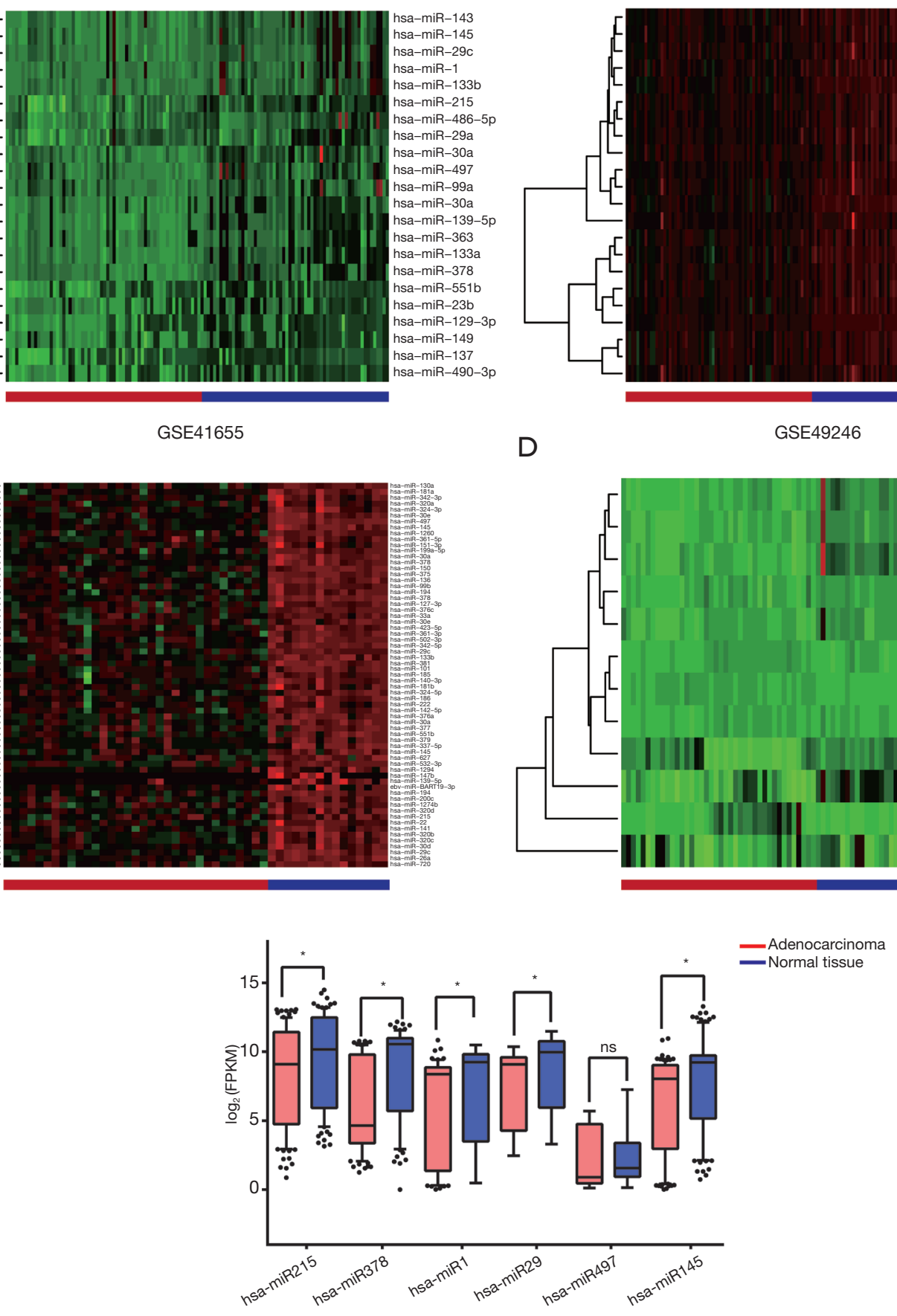

$\mathrm{D}$

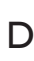

GSE38389

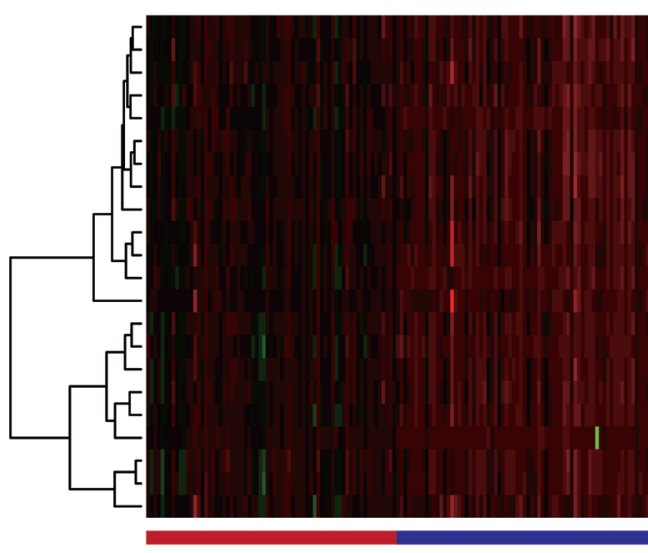

GSE49246

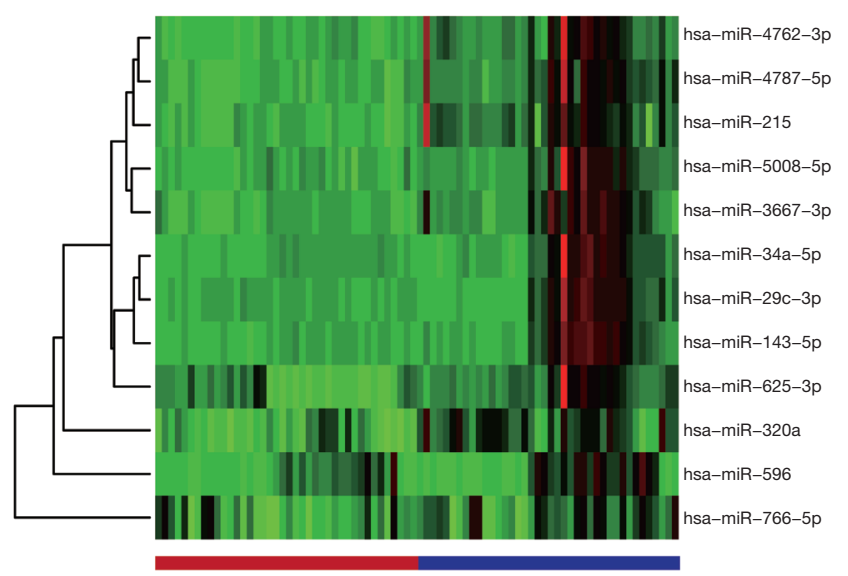

hsa-miR-342-3p hsa-miR-10b
hsa-miR-100 hsa-miR-338-3p hsa-miR-147b hsa-miR-192 hsa-miR-190 hsa-miR-30e hsa-let-7f hsa-miR-1 hsa-miR-145 hsa-miR-375 hsa-miR-133a hsa-miR-30c sa-miR-29c sa-miR-30b hsa-miR-195 ssa-miR-378 (s)-miR-215 sa-miR-192 - 1 R -143

E

Figure 1 miRNA expression in GSE48267 (A), GSE38389 (B), GSE41655 (C), GSE49246 (D) related to four pairs of CRC tissue samples and adjacent normal tissue. miRNAs that are generally underexpressed in 4 microRNA microarray $(\mathrm{E}) .{ }^{*}, \mathrm{P}<0.05$. 
A

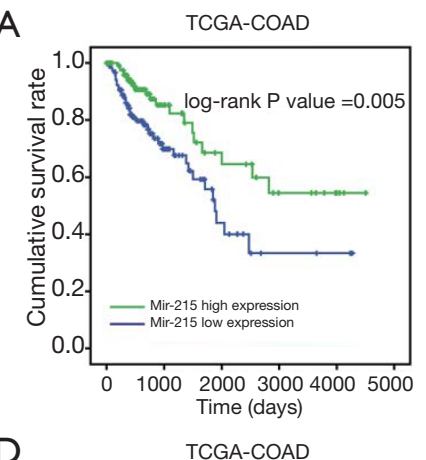

D

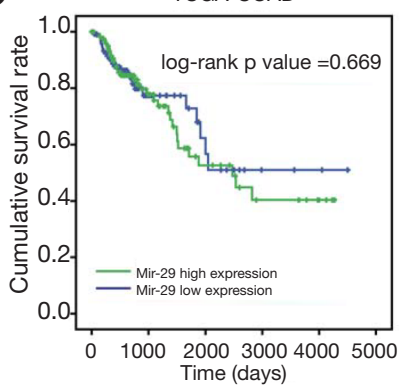

G

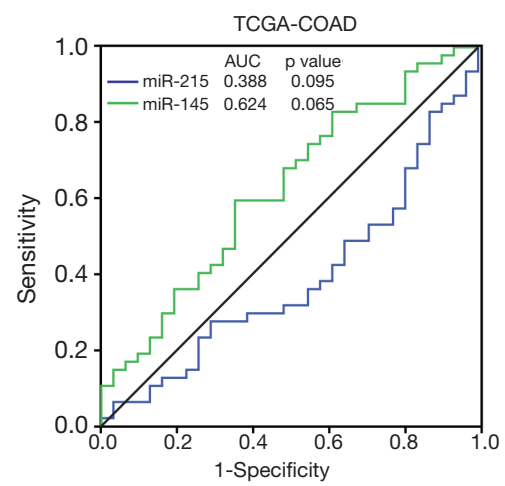

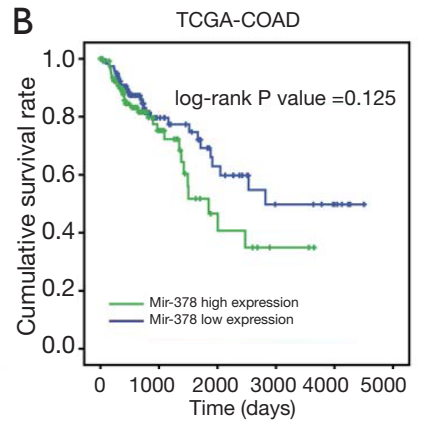
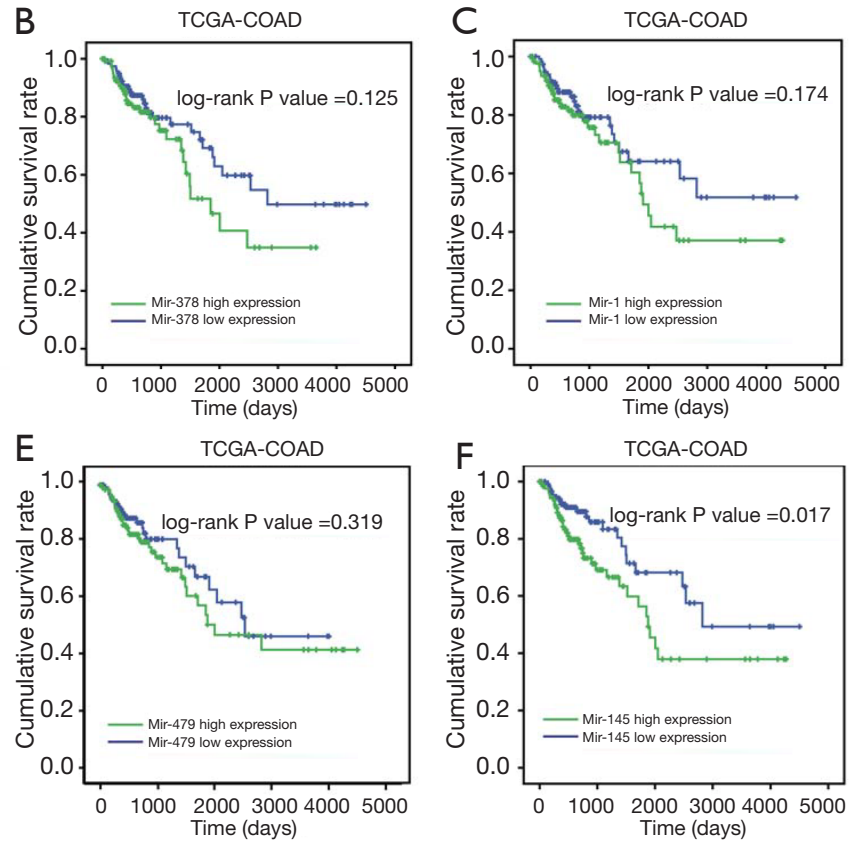

$\mathrm{H}$

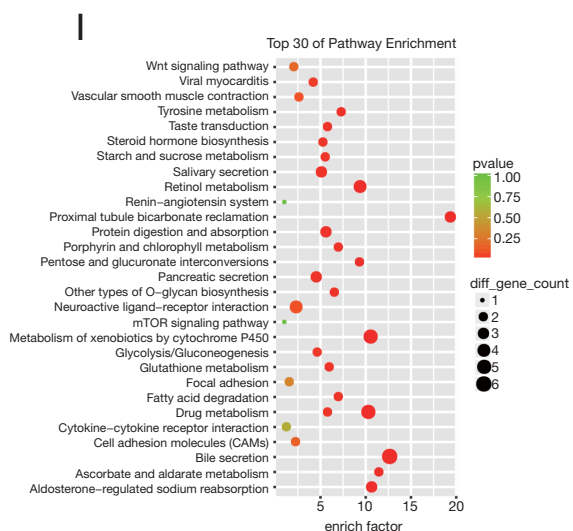

Figure 2 The expression of miR-215 in the TCGA database. Survival analysis of patients associated with a significant low expression of six miRNAs in tumor tissues in the TCGA-COAD cohort: miR-215 (A); miR-378 (B); miR-1 (C); miR-29 (D); miR-479 (E); miR-145 (F). The ROC curve predicted the 5-year survival in patients with low miR-215 and low miR-145 expression in the TCGA-COAD cohort (G). The survival curve of miR-215 in the tissue validation cohort collected in this study $(\mathrm{H})$. In the TCGA-COAD data set, KEGG predictive enrichment analysis of transcriptome differential genes was performed after grouping according to miR-215 expression (I). 
Table 2 Co-expression of miR-215, TRAF5 and IK $\beta$ - $\alpha$ in CRC tissue

\begin{tabular}{lccc}
\hline \multirow{2}{*}{ Related protein expression } & \multicolumn{2}{c}{ miR-215 expression, $\mathrm{n}(\%)$} & Chi-square \\
\cline { 2 - 3 } TRAF expression & High & & \\
High & $50(35.7)$ & $43(58.1)$ & 9.880 \\
Low & $90(64.3)$ & $31(41.9)$ & \\
IK $\beta$ - $\alpha$ expression & & $34(45.9)$ & $62.020^{\star}$ \\
High & $71(50.7)$ & $40(54.1)$ & $<0.001^{*}$ \\
Low & $69(49.3)$ &
\end{tabular}

*, significantly different by the Pearson chi-squared test, $\mathrm{P}<0.05$ was considered statistically significant.

hypothesis that miR-215 might affect TRAF5, thus interfering with the microenvironment of the CRC tumor. According to the TCGA-COAD database and our collection of tissue samples, patients with low expression of miR-215 had a worse prognosis (Figure $2 \mathrm{~A}$ and Figure $2 \mathrm{H}$ ). The prognosis of patients with high miR-215 expression combined with low TRAF5 protein expression was significantly worse than that of other subgroups with low miR-215 expression combined with low TRAF5 protein expression. The subgroup prognosis was also significantly worse than the prognosis in the subgroup of miR-215 with high TRAF5 protein expression, as shown in Figure $3 G$. Combining the patient's clinical and pathological parameters, a univariate and multivariate COX proportional risk analysis was performed, as shown in Table 3. miR-215 and TRAF5 protein expression was combined with the clinical and pathological parameters of the previous Cox regression model to establish a logistic regression model to predict the 5-year survival of CRC patients and to plot the ROC curve (Figure $3 H$ ). The area under the curve (AUC) was 0.741 , and the $\mathrm{P}$ value was $<0.01$, suggesting that the constructed multi-logistic diagnostic model had a very high predictive effect on the five-year survival of CRC patients (Figure 3G,H).

\section{Discussion}

Because of the advantages in their detection and stability, miRNAs are useful markers to evaluate tumor development, progression, and prognosis (23). However, the underlying potential mechanism resulting in the downregulation of miR-215 in tumors is still unknown. According to previous studies, miR-215 is downregulated in CRC tumor tissues compared to non-tumor tissues, suggesting its anti-oncogenic role (24). Indeed, miR-215 inhibits cell proliferation, invasion, and migration in several cell lines (25). However, the underlying potential mechanism resulting in the downregulation of miR-215 in tumors is still unknown.

In our present study, a number of GEO chip datasets were used and four miRNA chips were selected from a large sample size for integrated analysis. In our work, the low miR-215 expression was associated with poor prognosis in patients with CRC. This was also confirmed in our clinical specimen. Indeed, our results showed that low miR-215 expression in CRC patients was associated with TNM stage. After functional enrichment analysis and expression correlation of TCGA-COAD, the clinical expression relationship among miR-215, TRAF5 and IK $\beta-\alpha$ was analyzed, suggesting that miR-215 downregulation was associated with TRAF5 and IK $\beta-\alpha$ upregulation.

Many target genes involved in the above process were found as regulated by miR-215 (26). As a p53-responsive miRNA, miR-215 can enhance CDKN1A/p21 levels and suppress the expressions of denticleless protein homolog (DLT) and the activin receptor type 2B (ACVR2B) (27). Thus, our speculation is that miR-215 could take part in the immunopathological process in colon and rectal cancer through its effect on different target genes (28). Our established results on miR-215 combined with clinical pathological parameters of the logistics prediction model showed a good predictive role on the prognosis of CRC patients (29). Probably other mechanisms than the ones discovered in this study could be involved, but further studies are already planned to find potential target genes regulated by miR-215 and their significances in colon and rectal cancer.

Many miRNA are correlated with patients' survival. 
A

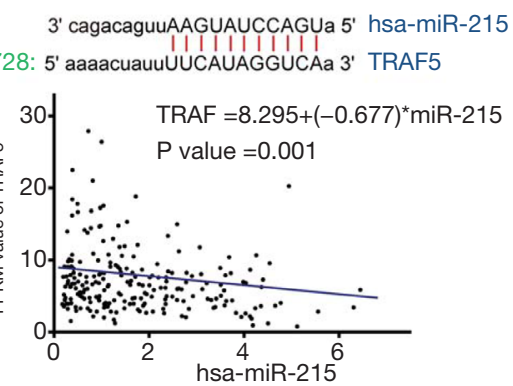

C

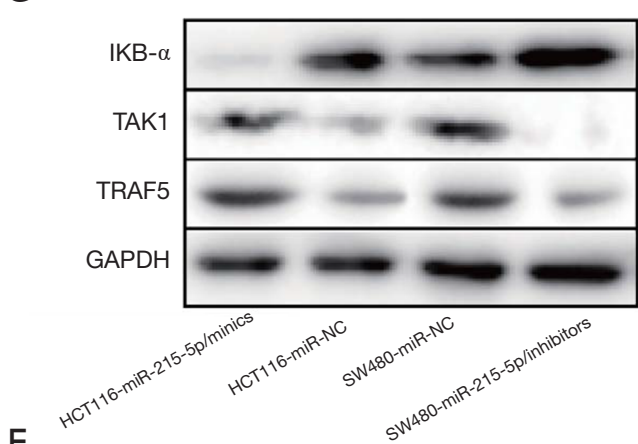

E

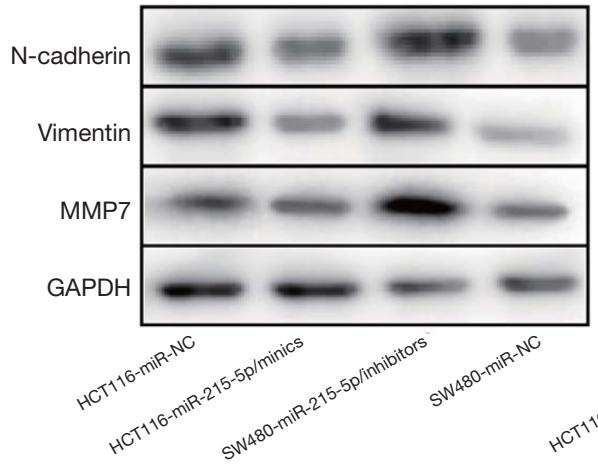

G

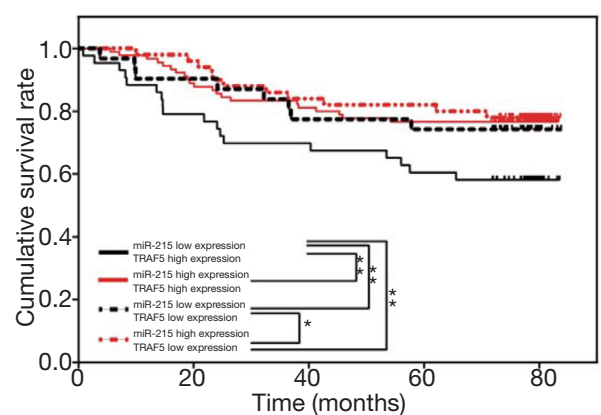

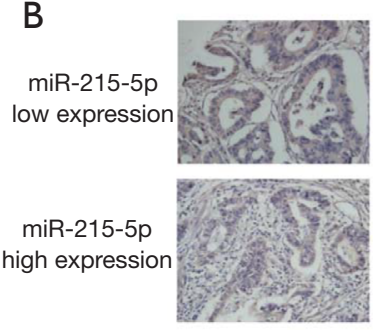

TRAF5

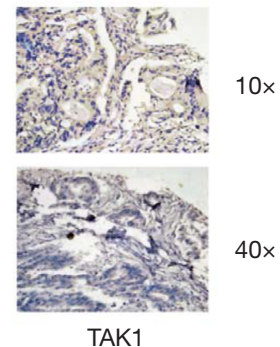

TAK1

D

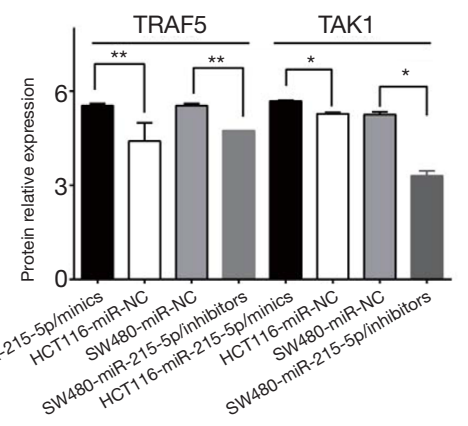

F

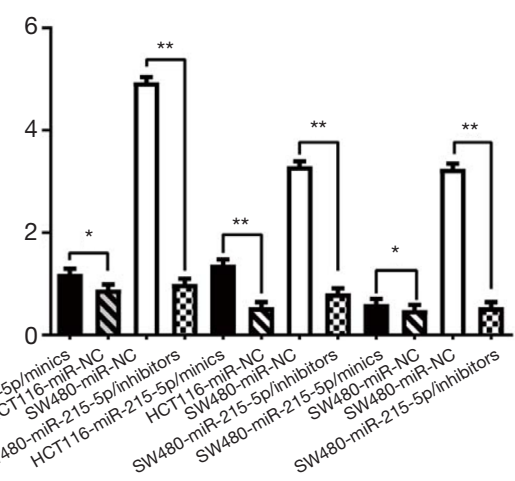

$\mathrm{H}$

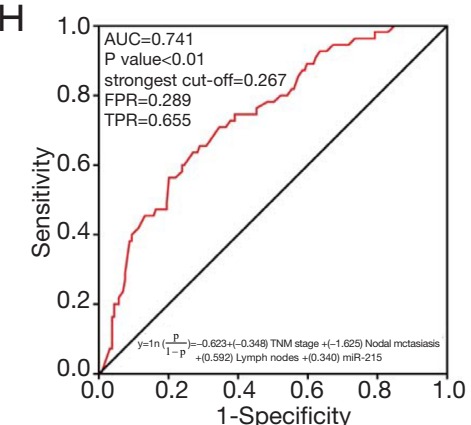

Figure 3 The expression of miR-215 in the TCGA database. Scatter plot of the correlation between miR-215 and TRAF5 expression in TCGACOAD data set (A). TRAF5 expression in CRC tumor specimens by immunohistochemical analysis using tissue microarray and TAK1 expression in CRC tumor specimens (B); Western blot analysis of TRAF, TAK1 and IK $\beta$ - $\alpha$ expression after miR-215-mimic and miR-215-inhibitor transfection into HCT116 and SW480 cells (C); Western blotting grayscale analysis of TRAF, TAK1 and IK $\beta$ - $\alpha$ expression after miR-215-mimic and miR-215-inhibitor transfection into HCT116 and SW480 cells (D). Western blot analysis of MMP7, vimentin and N-cadherin expression after miR-215-mimic and miR-215-inhibitor transfection into HCT116 and SW480 cells (E,F). Kaplan-Meier curves showing the impact of miR215 and TRAF5 expression subgroup for overall survival of 214 CRC patients (G). ROC curve determining the performance of the multivariate regression prediction model on the prognosis of the 214 patients with $\mathrm{CRC}(\mathrm{H})$. $^{*}, \mathrm{P}<0.05 ;{ }^{* *}, \mathrm{P}<0.01$. 
Table 3 Univariate and multivariate Cox regression

\begin{tabular}{|c|c|c|c|c|}
\hline Clinical parameters & \multicolumn{2}{|c|}{ Univariate analysis } & \multicolumn{2}{|c|}{ Multivariate analysis } \\
\hline Gender (female vs. male) & $1.08(0.65-1.81)$ & 0.770 & & \\
\hline Age ( $\geq 63$ vs. <63) & $0.71(0.42-1.19)$ & 0.187 & & \\
\hline Tumor location (rectum vs. colon) & $1.50(0.88-2.55)$ & 0.134 & & \\
\hline Tumor (T) status ( $\mathrm{T} 3+\mathrm{T} 4$ vs. $\mathrm{T} 1+\mathrm{T} 2)$ & $0.32(0.18-0.56)$ & 0.099 & & \\
\hline Nodal (N) metastasis (N1+N2 vs. N0) & $1.69(0.86-3.33)$ & $<0.01^{*}$ & $3.72(1.52-9.14)$ & $0.004^{*}$ \\
\hline Lymph nodes ( $\geq 12$ vs. $<12$ ) & $2.05(1.22-3.42)$ & $0.007^{*}$ & $0.67(0.28-1.62)$ & 0.373 \\
\hline miR-215 (high vs. low) & $0.59(0.35-0.91)$ & $0.046^{*}$ & $0.61(0.36-1.03)$ & 0.064 \\
\hline
\end{tabular}

${ }^{*}, \mathrm{P}<0.05$ was considered statistically significant.

For instance, patients with high miR-21 expression have less recurrence-free cancer-specific survival in stage II colon cancer. In addition, high miR-29a or miR-362-3p expression is associated with a longer disease-free survival, and miR-93 can inhibit the early relapse of CRC. Our study showed that miR-215 had an effect on TRAF5 and TAK1. Possible effects on ubiquitination degradation of IK $\beta-\alpha$, and then activation of the NF- $\mathrm{KB}$ signaling pathway promote inflammation-promoting tumor progression (30). However, in our study, no significant difference in the linear models of miR-215 and TRAF5 expression and miR-215 and IK $\beta-\alpha$ expression was found. Our hypothesis is that the mall sample size could have a negative influence in the significance of our results (31), together with the use of TRAF5 and IK $\beta-\alpha$ protein expression by immunohistochemical techniques, subject to our collection of paraffin tissue specimens. Therefore, more in-depth research will be performed using more samples of high-throughput sequencing combine algorithm models with higher fitness.

\section{Conclusions}

An integrated analysis of miRNAs was performed on human normal colorectal epithelial tissue and colorectal adenocarcinoma tissues, providing a rich resource for exploring the role of several miRNAs in CRC. miR-215 might be a potentially important biomarker in the CRC diagnosis and prognosis. miR-215 was downregulated in CRC tissues from patients with poor prognosis compared with its overexpression resulting in a better prognosis. Moreover, in our validation experiment performed in cell lines, miR-215 downregulation was accompanied by a significant upregulation of TRAF5 and IK $\beta-\alpha$ expression. Therefore, our results might be useful in the association of miR-215/TRAF5 interaction in CRC patients and their poor prognosis.

\section{Acknowledgments}

Funding: This work was supported by the National Natural Science Foundation of China (no. 81372375). The grant from Science and Technology Development Medical and Health Guidance Plan Project of Wuxi City (no. NZ2019002). The grant from Fundamental Research Funds for the Central Universities funded by the Ministry of Education of China (no. JUSRP51710A).

\section{Footnote}

Conflicts of Interest: All authors have completed the ICMJE uniform disclosure form (available at http://dx.doi. org/10.21037/tcr-19-2424). The authors have no conflicts of interest to declare.

Ethical Statement: The authors are accountable for all aspects of the work in ensuring that questions related to the accuracy or integrity of any part of the work are appropriately investigated and resolved. All patients enrolled 
in this study provided written informed consent to the use of their tissues, and this study was approved by the Ethics Committee Affiliated of Hospital of Jiangnan University, China (No. 20180642). The study was conducted in accordance with the Declaration of Helsinki (as revised in 2013).

Open Access Statement: This is an Open Access article distributed in accordance with the Creative Commons Attribution-NonCommercial-NoDerivs 4.0 International License (CC BY-NC-ND 4.0), which permits the noncommercial replication and distribution of the article with the strict proviso that no changes or edits are made and the original work is properly cited (including links to both the formal publication through the relevant DOI and the license). See: https://creativecommons.org/licenses/by-nc-nd/4.0/.

\section{References}

1. Bye WA, Ma C, Nguyen TM, et al. Strategies for Detecting Colorectal Cancer in Patients with Inflammatory Bowel Disease: A Cochrane Systematic Review and MetaAnalysis. Am J Gastroenterol 2018;113:1801-9.

2. Clarke WT, Feuerstein JD. After surgery for stage II or III colorectal cancer, more vs less frequent followup did not differ for 5-year mortality. Ann Intern Med 2018;169:JC38.

3. Liang Q, Ma D, Zhu X, et al. RING-finger protein 6 amplification activates JAK/STAT3 pathway by modifying SHP-1 ubiquitylation and associates with poor outcome in colorectal cancer. Clin Cancer Res 2018;24:1473-85.

4. Lee J, Jin YA, Ko HY, et al. Magnetic resonance beacon to detect intracellular microRNA during neurogenesis. Biomaterials 2015;41:69-78.

5. He C, Yu T, Shi Y, et al. MicroRNA 301A Promotes Intestinal Inflammation and Colitis-Associated Cancer Development by Inhibiting BTG1. Gastroenterology 2017;152:1434-48.e15.

6. Yuan C, Burns MB, Subramanian S, et al. Interaction between Host MicroRNAs and the Gut Microbiota in Colorectal Cancer. mSystems 2018;3:e00205-17.

7. Zhang Y, Guo L, Li Y, et al. MicroRNA-494 promotes cancer progression and targets adenomatous polyposis coli in colorectal cancer. Mol Cancer 2018;17:1.

8. Ren Y, Shang J, Li J, et al. The long noncoding RNA PCAT-1 links the microRNA miR-215 to oncogene CRKL-mediated signaling in hepatocellular carcinoma. J Biol Chem 2017;292:17939-49.
9. Kori M, Arga KY, et al. Potential biomarkers and therapeutic targets in cervical cancer: Insights from the meta-analysis of transcriptomics data within network biomedicine perspective. PLoS One 2018;13:e200717.

10. Ullmann P, Nurmik M, Schmitz M, et al. Tumor suppressor miR-215 counteracts hypoxia-induced colon cancer stem cell activity. Cancer Lett 2019;450:32-41.

11. Agostini A, Brunetti M, Davidson B, et al. The microRNA miR-192/215 family is upregulated in mucinous ovarian carcinomas. Sci Rep 2018;8:11069.

12. Wei Y, Sun J, Li X, et al. MicroRNA-215 enhances invasion and migration by targeting retinoblastoma tumor suppressor gene 1 in high-grade glioma. Biotechnol Lett 2017;39:197-205.

13. Wang YX, Zhang TJ, Yang DQ, et al. Reduced miR-215 expression predicts poor prognosis in patients with acute myeloid leukemia. Jpn J Clin Oncol 2016;46:350-6.

14. Sun M, Song H, Wang S, et al. Integrated analysis identifies microRNA-195 as a suppressor of Hippo-YAP pathway in colorectal cancer. J Hematol Oncol 2017;10:79.

15. Li N, Li Y, Li Z, et al. Hypoxia Inducible Factor 1 (HIF1) Recruits Macrophage to Activate Pancreatic Stellate Cells in Pancreatic Ductal Adenocarcinoma. Int J Mol Sci 2016;17:799.

16. Wang Y, Xue D, Li Y, et al. The Long Noncoding RNA MALAT-1 is A Novel Biomarker in Various Cancers: A Meta-analysis Based on the GEO Database and Literature. J Cancer 2016;7:991-1001.

17. Pashaei E, Guzel E, Ozgurses ME, et al. A Meta-Analysis: Identification of Common Mir-145 Target Genes that have Similar Behavior in Different GEO Datasets. PLoS One 2016;11:e0161491.

18. Chen WJ, Tang RX, He RQ, et al. Clinical roles of the aberrantly expressed lncRNAs in lung squamous cell carcinoma: A study based on RNA sequencing and microarray data mining. Oncotarget 2017;8:61282-304.

19. Gray RT, Cantwell MM, Coleman HG, et al. Evaluation of PTGS2 Expression, PIK3CA Mutation, Aspirin Use and Colon Cancer Survival in a Population-Based Cohort Study. Clin Transl Gastroenterol 2017;8:e91.

20. Muhammad S, Tang Q, Wei L, et al. miRNA 30d serves a critical function in colorectal cancer initiation, progression and invasion via directly targeting the GNA13 gene. Exp Ther Med 2019;17:260-72.

21. Mallona I, Sierco A, Peinado MA, et al. The Pancancer DNA Methylation Trackhub: A Window to The Cancer Genome Atlas Epigenomics Data. Methods Mol Biol 2018;1766:123-35. 
22. Wang F, Wu J, Qiu Z, et al. ACOT1 expression is associated with poor prognosis in gastric adenocarcinoma. Hum Pathol 2018;77:35-44.

23. Li J, Kong C, Liu Q, et al. Colorimetric ultrasensitive detection of DNA based on the intensity of gold nanoparticles with dark-field microscopy. Analyst 2018;143:4051-6.

24. Vychytilova-Faltejskova P, Slaby O. MicroRNA-215: From biology to theranostic applications. Mol Aspects Med 2019;70:72-89.

25. Wang T, Chen N, Ren W, et al. Integrated analysis of circRNAs and mRNAs expression profile revealed the involvement of hsa_circ_0007919 in the pathogenesis of ulcerative colitis. J Gastroenterol 2019;54:804-18.

26. Ullmann P, Nurmik M, Schmitz M, et al. Tumor suppressor miR-215 counteracts hypoxia-induced colon cancer stem cell activity. Cancer Lett 2019;450:32-41.

27. Rau CS, Wu SC, Yang JCS, et al. Profiling the circulating miRNAs in mice exposed to gram-positive and gram-

Cite this article as: Wang Z, Jiang $\mathrm{X}$, Li Q, Jin Y, Liu X, Wang F, Mao Y, Hua D. Integrated analysis identifies low microRNA-215 expression as associated with a poor prognosis of patients with colorectal cancer through the IK $\beta$ - $\alpha$ signaling pathway. Transl Cancer Res 2020;9(9):5233-5244. doi: 10.21037/ tcr-19-2424 negative bacteria by Illumina small RNA deep sequencing. J Biomed Sci 2015;22:1.

28. Magga J, Vainio L, Kilpiö T, et al. Systemic Blockade of ACVR2B Ligands Protects Myocardium from Acute Ischemia-Reperfusion Injury. Mol Ther 2019;27:600-10.

29. González-Sarrías A, Núñez-Sánchez MA, Tomé-Carneiro $\mathrm{J}$, et al. Comprehensive characterization of the effects of ellagic acid and urolithins on colorectal cancer and keyassociated molecular hallmarks: MicroRNA cell specific induction of CDKN1A (p21) as a common mechanism involved. Mol Nutr Food Res 2016;60:701-16.

30. Sebio A, Gerger A, Matsusaka S, et al. Genetic variants within obesity-related genes are associated with tumor recurrence in patients with stages II/III colon cancer. Pharmacogenet Genomics 2015;25:30-7.

31. Huang YQ, Liang CH, He L, et al. Development and Validation of a Radiomics Nomogram for Preoperative Prediction of Lymph Node Metastasis in Colorectal Cancer. J Clin Oncol 2016;34:2157-64. 